\title{
Intelligent machine for sorting semi-precious minerals
}

\author{
Mikhail Polishchuk ${ }^{1}$, Mikhail Tkach ${ }^{2}$, Igor Parkhomey ${ }^{3}$, Juliy Boiko ${ }^{4}$, \\ Yevhenii Batrak ${ }^{5}$, Oleksander Eromenko ${ }^{6}$ \\ 1,2,3,5 Department of Technical Cybernetics, National Technical University of Ukraine \\ "Igor Sikorsky Kyiv Polytechnic Institute", Ukraine \\ ${ }^{4}$ Department of Telecommunications and Radio Engineering, Khmelnytsky National University, Ukraine \\ ${ }^{6}$ Department of Physics and Electrical Engineering, Khmelnytsky National University, Ukraine
}

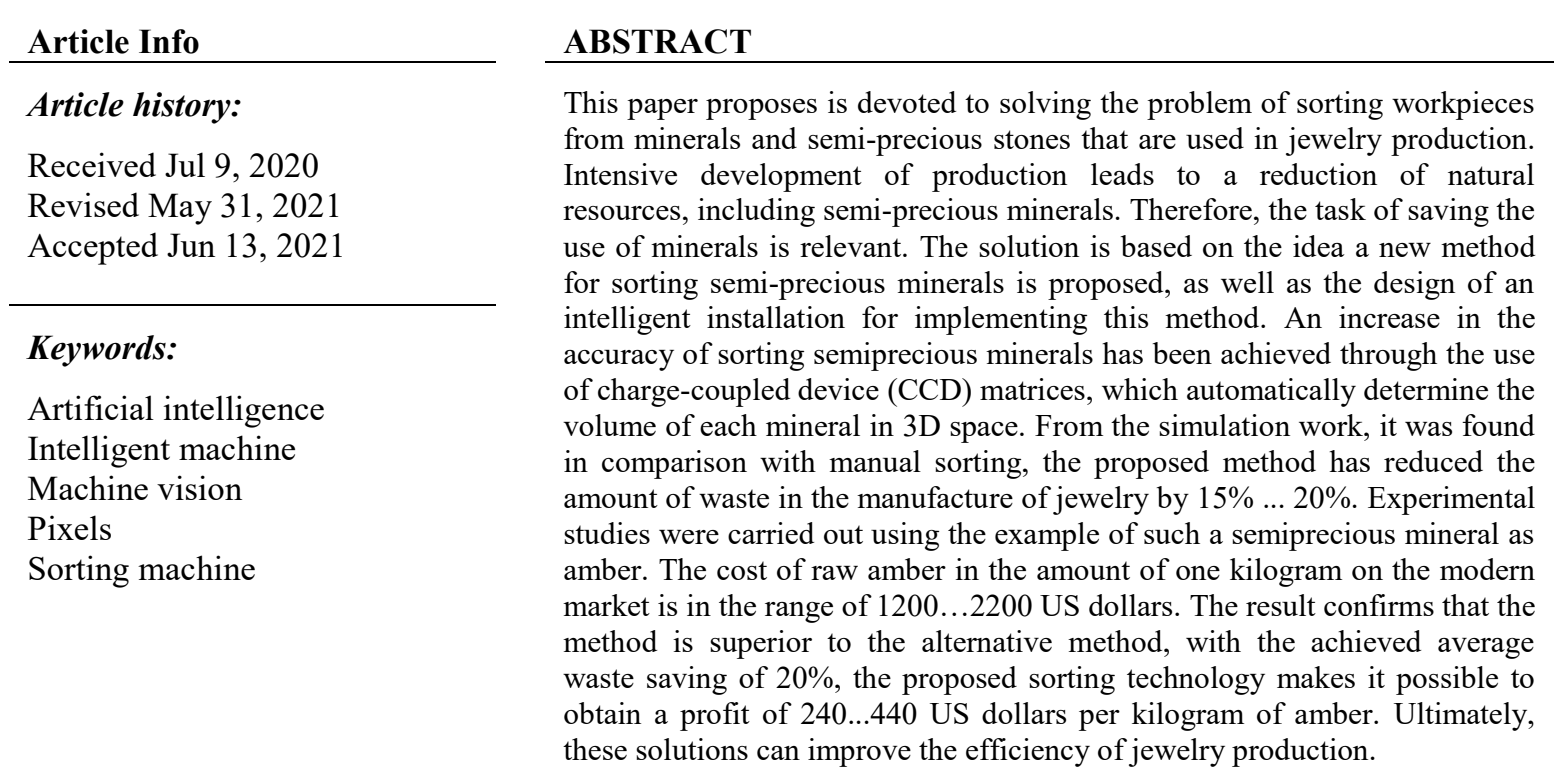

This is an open access article under the CC BY-SA license.

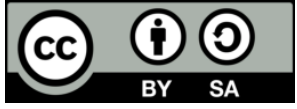

\section{Corresponding Author:}

Juliy Boiko

Department of Telecommunications and Radio Engineering

Khmelnytsky National University

11 Instytuts'ka St., Khmelnitsky, 29016, Ukraine

Email: boiko_julius@ukr.net

\section{INTRODUCTION}

In many industries, there are processes for sorting minerals with the goal of dividing them into groups according to the volume, overall dimensions, the color, and even chemical composition. In this case, various optical, electronic and radio engineering systems with program control are used. The most famous systems of machine and computer vision with digital image processing [1]-[3]. However, in these papers are set forth general technologies for recognizing objects without solving the problem of sorting minerals by any signs.

In [4], [5], an overview of the choice of industrial video cameras for machine vision systems, which are best suited for creating mineral sorting plants, is proposed. However, this paper does not propose algorithms for recognizing sorting objects. A known method [6] for determining the granular composition of crushed rock in quarries. According to this method, the rock mass is photographed at any angle and, on the basis of a computer program, the rock pieces are separated into particle size classes. This method allows to 
increase the accuracy of determining the granular composition of the rock in the total mass, however, it does not make it possible to sort each stone separately, which is its drawback.

In the method [7], [8] when sorting piece goods, the selection criterion is the minimum of space that remains free after cargo is placed. If we use the analogy of comparing the volume of the mineral stock and the finished product, this method can be adapted for sorting semiprecious stones. An example of a mechanical sorting of minerals is the device [9], [10] for sorting solid materials by size. But this device has a low sorting accuracy, which is not applicable for precious minerals. An interesting example is the contactless sorting of products using video cameras [11]-[14] and the processing of the resulting images for sorting by the average diameter of the products.

This restriction is acceptable for sorting fruits, but not acceptable for precious and semi-precious minerals due to the low accuracy of sorting. For example, in agriculture, a promising area for product sorting systems is the use of mobile robots [15], [16] as actuators. But unfortunately, these robots are in lack of optical recognition systems. This problem can be solved by applying research [17], [18] in the field of machine and artificial intelligence, as well as using algorithms for detecting objects on a humanoid robot [19], [20]. Thus, the problem of sorting semi - precious minerals with the goal of reducing waste of precious raw materials remains relevant.

Unlike studies [4], [5], this work is the first to propose sorting minerals according to such features as the gross volume of raw materials and the net volume of the future jewelry. In contrast to technical solutions [5]-[10], this study for the first time proposed the idea is based to use the difference between the above gross volume of raw materials and the net volume of jewelry as a sorting criterion. As the experimental studies outlined have shown, the proposed sorting method makes it possible to save up to $20 \%$ of the initial volume of minerals in waste of semi-precious raw materials. Also proposed is a patented design [18] of a fundamentally new intelligent machine for sorting minerals. The design of the new machine is distinguished by the presence of three charge-coupled device (CCD) matrices for identifying volumes of minerals in a $3 \mathrm{D}$ coordinate system, as well as automatic devices for distributing minerals into individual containers, depending on the volumes of semi-precious mineral stones.

\section{RESEARCH METHOD}

The method is based on the use of charge-coupled device (CCD) [21], [22] matrices with a specific resolution, measured in pixels (pixel-picture element) per inch (ppi-pixels per inch). The essence of the method lies on the fact that initially the gross volume of the mineral arriving for sorting is determined, and then it is compared with one or more net volumes of manufactured products and then the sorted mineral is sent to containers that correspond to the minimum difference between the specified gross volume of the workpiece and the net volumes of products. This method allows us to minimize the waste of semi-precious and precious minerals in the manufacture of jewelry from them with specified dimensional parameters.

\subsection{Solution of the problem under consideration}

The method and design of the apparatus for sorting small sized workpieces from minerals and semiprecious stones proposed can reduce the amount of waste in the process of further mechanical processing of minerals for the purpose of manufacturing jewelry [22], [23]. The proposed method for sorting minerals is aimed at increasing the volume of finished jewelry from one mineral. The technical solutions described can be used to increase the efficiency of the jewelry industry in the manufacture of semiprecious and precious stones, such as amber, emeralds, garnets, topazes, Icelandic spar, rock crystal and other semiprecious minerals as shown in Figure 1.
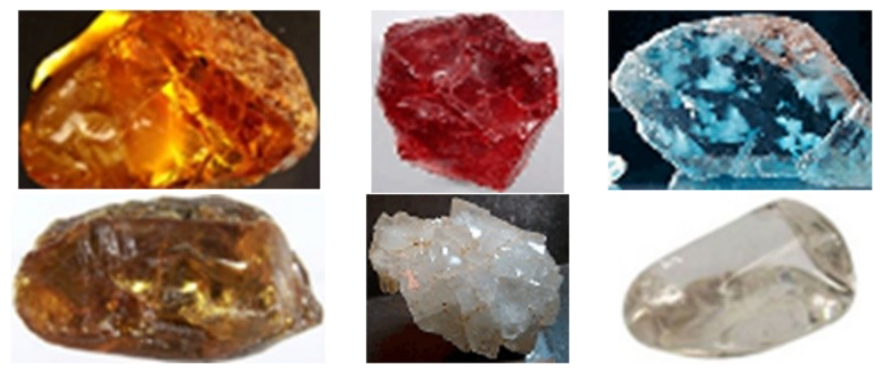

Figure 1. Examples of semiprecious minerals 


\subsection{The method of minerals recognition}

We will consider the sorting method by the example of making jewelry in the form of beads [18], for example, for beads, necklaces, bracelets, and similar jewelry. The task is to place the maximum number of future products, for example, spherical or other, in each mineral stone. Then, when billets from minerals are sawing, it seems possible to ensure the minimum amount of waste of semi - precious mineral.

In Figure 2 is shown conventionally three CCD matrices of an analog integrated circuit consisting of photosensitive photodiodes. Based on such matrices, modern video cameras are based. The matrices shown in Figure 2. Namely: Figures 2 (a) "ZX", (b) "ZY", (c) "XY", are located in the corresponding planes of the Cartesian coordinate system XYZ and have a physical size $\mathrm{A} \times \mathrm{B}$ with a pixel size of "c" and their number (cells) for each coordinate from 1, 2, $3 \ldots$ to " $n "$. Matrices are characterized by a certain resolution, measured in pixels (pixel-Picture Element) per inch (ppi-pixels per inch). In accordance with the proposed method for sorting minerals, technological operations are performed in the following sequence in steps [24].

Step 1: The next mineral to be sorted and shown in Figure 2 (d), serves in the video review area of the three cameras. At this step, three projections of the mineral are taken: (a) frontal, (b) profile, and (c) plan view in the cartesian coordinate system Figure (d) "XYZ". The area of each projection is limited by the contours of $\mathrm{F}_{\mathrm{zx}}, \mathrm{F}_{\mathrm{zy}}, \mathrm{F}_{\mathrm{xy}}$ images having the corresponding sizes $\mathrm{H}_{\mathrm{z}} \times \mathrm{L}_{\mathrm{x}}, \mathrm{Hz} \times \mathrm{B}_{\mathrm{y}}$ and $\mathrm{B}_{\mathrm{y}} \times \mathrm{L}_{\mathrm{x}}$.

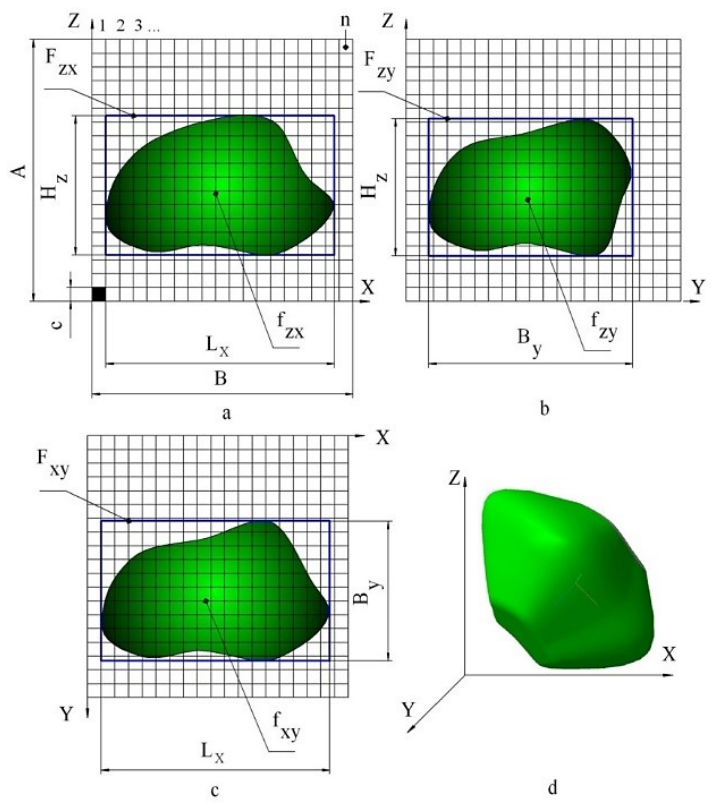

Figure 2. Arrangement of three CCD-matrices in the planes of the XYZ coordinate system:

(a) in coordinates "ZY", (b) in coordinates "XY", (c) in coordinates "XYZ"

Step 2: Using the processor of the control unit, the number of pixels contained in the projection loop of the mineral itself is calculated on each of the three CCD matrices of the respective cameras. The number of pixels per region bounded by the outline of the mineral projection is the area of the corresponding projection, namely $\mathrm{f}_{\mathrm{zx}}, \mathrm{f}_{\mathrm{zy}}$ and $\mathrm{f}_{\mathrm{xy}}$.

Step 3: Then, determine the gross volumes of the mineral in the direction of the corresponding coordinate axes, namely: $V_{1}=f_{z x} \times B_{y} ; V_{2}=f_{z y} \times L_{x} ; V_{2}=f_{x y} \times H_{z}$. Knowing the resolution value of the video camera, all measurement results in pixels can be converted into metric values. So, with the equality of $1 \mathrm{inch}=25.4 \mathrm{~mm}$, according to the formula $25.4 / \delta$, where is the resolution of the video camera, we can get the pixel size, and therefore all measurements in $\mathrm{mm}$, which is needed when comparing the gross volumes of the mineral with the actual volumes of manufactured products. The gross volumes obtained at this step of the method implementation include cells (pixels) of the space of not only of the mineral body, but also of the image cell in the direction of the corresponding coordinate axes. This error for industrial sorting is not significant, because modern camcorders with $1 / 4$-inch matrices and resolutions up to $1200 \times 720$ have a physical pixel size from $0.005 \mathrm{~mm}$ to $0.009 \mathrm{~mm}$. Given that from the point of view of industrial feasibility, the size range of performs of small-sized minerals is in the range from $5 \mathrm{~mm}$ to $35 \mathrm{~mm}$, the indicated error in determining the gross volume of the mineral will not exceed $1 \%$ of the actual volume of the mineral. 
Step 4: After all, choose the minimum volume $\mathrm{V}_{\min }$ from the obtained three gross volumes and compare it with the known values of the volumes of future products or semi-finished products, as shown in Figure 3. Future products, as a rule, have the correct shape, for example, a ball, hexagonal prism, rhombohedra, cube and the like, depending on the type of cut of the product from the mineral. For a better understanding of the method, as an example, consider a special case of the manufacture of balls of net volumes $V_{i}$ and the radius $R_{i}$ for a necklace or amber bracelet, where " $i$ " is the number of the ball, and its volume, as know, is equal $\mathrm{V}_{\mathrm{i}}=4 / 3 \times \pi \mathrm{R}_{\mathrm{i}}{ }^{3}$. As can be seen from Figures 3 (a), (b), (c) from the same mineral it is possible to produce a different number of balls with different diameters. As a criterion for rational choice according to this method take the minimum difference in volume $\mathrm{V}_{\min }$ mineral and volume of the future product, i.e. ball $\mathrm{V}_{\mathrm{i}}$, namely: $\mathrm{V}_{\min }-\mathrm{V}_{\mathrm{i}}=\Delta_{\min }$, where $\Delta_{\min }$ - the minimum amount of inevitable waste of raw materials. The possible number of balls is determined by the minimum difference in the projections of the areas $f_{\min }$ mineral and ball $f_{i}=\pi \times R_{i}^{2}$, i.e: $f_{\min }-\pi R_{i}^{2}=\delta_{\min }$, where $\delta_{\min }$ - the minimum part of the mineral projection area remaining after applying a different number of balls. It is obvious that when determining $\delta_{\text {min }}$, in the case of two balls as shown in Figure 3 (b), the cutting plane of the mineral will pass along section $\mathrm{A}-\mathrm{A}$, and in the case of manufacturing three balls, the cutting of the mineral will be carried out along sections $\mathrm{B}-\mathrm{B}$ and $\mathrm{C}-\mathrm{C}$ as shown in Figure 3 (c).

Step 5: Based on the results of selecting the minimum amount of valuable raw material waste and determining the rational amount of future products from one mineral, this mineral is sent to a container that corresponds to the minimum difference between the indicated gross volume of the work piece and the net volume of products. Thus, in contrast to the analogs mentioned above in section 1. Introduction, the accuracy of measuring the volumes of minerals has been increased to one pixel of the CCD matrix, namely, to $0.15 \ldots 0.20 \mathrm{~mm}$.

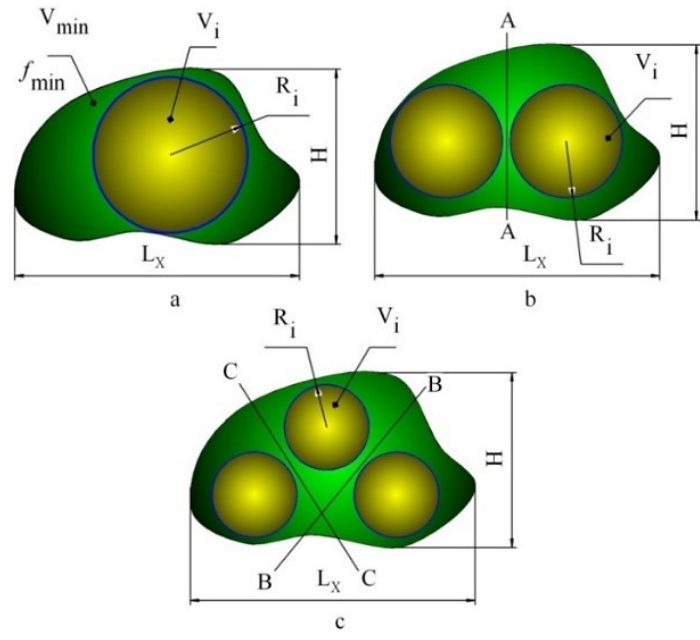

Figure 3. Determining the possible number of balls in one mineral object to calculate the minimum amount of waste: (a) the case of one ball, (b) the case of two balls, (c) the case with three balls

\subsection{Mineral sorting machine design}

In Figure 4 is shown a general view of a plant for sorting semi - precious minerals. After loading the minerals into the vibrating hopper 1 and turning it on, mineral blanks under the influence of vibrations in a single layer flow move along the spiral paths 2 and go to the tray 3 . The speed of the conveyor 4 is several times higher than the feed speed of the single layer blanks of the vibratory hopper 1 . Therefore, when coming from the tray 3 of one of the minerals to the conveyor 4, a separate mineral is rapidly separated from the mineral flow, which is supplied by the hopper 1 .

At the signal of the photo sensor 5 , the interrupter 6 stops the flow, and the mineral located on the conveyor 4 enters the field of view of the cameras 7 . These cameras take pictures of three mineral projections according to the above method. Moreover, when taking mineral pictures, the conveyor stop is not required, because the speed of modern video cameras based on chained diagonally dominant (CDD)-matrices is at least 25 frames per second, which is an order of magnitude faster than the conveyor speed. Next, the mineral that has passed the video surveillance zone of the cameras 7, through the tray 8 enters the distribution tray 9 . This will be reported to the control system by the photo sensor 10 signal. The operation of this sensor is the basis for supplying control [25] action from the controller 11 to raise the chopper 6 of the workpiece stream and pass the next mineral onto conveyor 4 . At the same time, the distribution tray 9 with the mineral in it is 
rotated by a certain angle, which determines its orientation relative to a particular storage container 12 . The rotation angle of the distribution tray 9 is determined by the controller 11 command, which in turn is formed according to the measurement results according to the above sorting method, which determines $d_{i}$ as shown in Figure 4 sizes and the amount of $\mathrm{N}_{\mathrm{i}}$ of future products, taking into account the minimum amount of waste of valuable raw materials. In addition to those indicated in Figure 4 components, the installation contains a control panel 13, a power supply 14, a compressed air preparation unit 15 and a compressed air distributor 16 for pneumatic drives.

Thus, the fundamental difference of this machine from the analogs mentioned above is a set of CCD matrices as shown in pos. 7, Figure 4 in 3D space and a controller (pos. 13), which calculates the volume of each mineral according to the algorithm of the program presented in section 2.2. The method of minerals recognition.

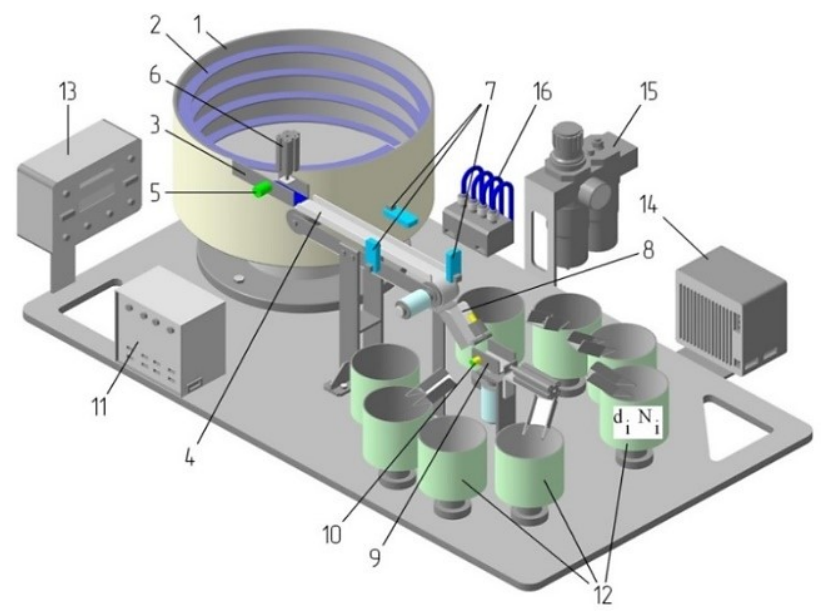

Figure 4. Sorting machine for semi-precious mineral

\subsection{Experimental studies of mineral sorting}

The purpose of the experiment is to determine the effect and volume of the mineral stones of such parameters as its length, width and height. In the case of convergence of these parameters, there will be a minimum of waste in the processing of stones of semi-precious minerals. Amber, a popular mineral, was chosen as the object of the experiment. At the first stage, amber minerals were distributed in shape and size into 7 groups as shown in Figure 5, lengths from $5 \mathrm{~mm}$ to $35 \mathrm{~mm}$, in increments of $5 \mathrm{~mm}$. At the second stage, each group passed the weighing of the amber group and counting their number. The results of these measurements are shown in Table 1.

According to the results of the obtained statistical data, it can be concluded that the most frequently encountered samples are stones from $10 \mathrm{~mm}$ to $20 \mathrm{~mm}$ long. Therefore, these parameter values are taken as limitations for varying the limiting sizes of minerals. The forms of semi-precious minerals are various and probabilistic. Therefore, the experiment should determine the effect of geometric parameters (length, width, height) of minerals on the volume of stones, such as amber. The solution of this problem will increase the efficiency of sorting of semi-precious minerals in order to reduce waste during their machining in the process of making jewelry.

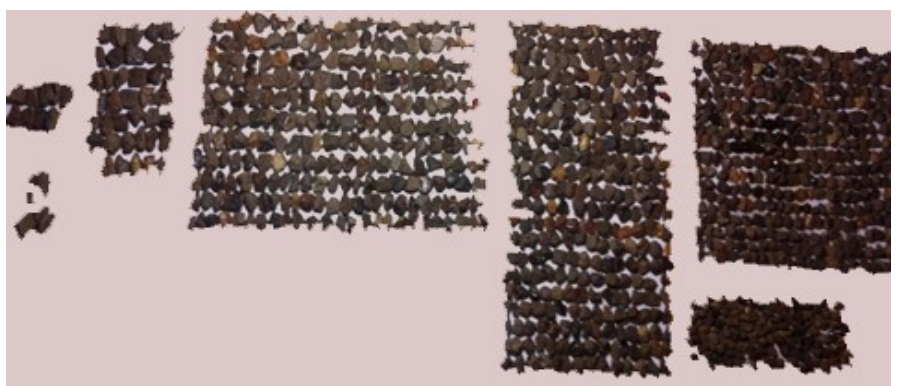

Figure 5. Preliminary division of amber stones into 7 groups 
Table 1. Amber mineral groups

\begin{tabular}{ccccc}
\hline Group number & Length $(\mathrm{mm})$ & Weight $($ grams $)$ & Amount & Percentage \% of the total \\
\hline 1 & 5 & 56 & 126 & 11,6 \\
2 & 10 & 238 & 363 & 33.5 \\
3 & 15 & 298 & 293 & 26,9 \\
4 & 20 & 334 & 249 & 22,9 \\
5 & 25 & 68 & 41 & 3,8 \\
6 & 30 & 22 & 10 & 0,9 \\
7 & 35 & 12 & 4 & 0,4 \\
& Total: & & 1086 & $100 \%$ \\
\hline
\end{tabular}

As a hypothesis, which requires further confirmation, at the stage of problem statement, we present the objective function in the form of a formula,

$$
V=C_{0} f_{1}(L) f_{2}(B) f_{3}(H) \rightarrow \max
$$

with restrictions:

$$
L_{\min }<L_{i} \leq L_{\max } ; B_{\min } \leq D_{i} \leq B_{\max } ; H_{\min } \leq H_{i} \leq H_{\max }
$$

where: $V$-volume of mineral, $\mathrm{m}^{3} ; C_{0}$-a constant coefficient taking into account the influence of external influences that are not displayed in the objective function; $f_{1}(L), f_{2}(B), f_{3}(H)$-factor dependencies (variable functions) to be determined; $L, B, H$-respectively the length, width and height of the mineral $(\mathrm{mm})$. In accordance with the accepted restrictions (2) in the formulation of a full-factor experiment, the variation of the factors of the objective function was carried out within the following limits.

$$
5<L_{i} \leq 20 ; 5 \leq B_{i} \leq 20 ; 5 \leq H_{i} \leq 20
$$

In total, experiments were conducted $N=n^{\cdot k} m=2^{3} \cdot 3=24$, where: $\mathrm{n}$ is the number of levels of factor variation ( $\max , \min )$; $k$-is the number of factors; $m$-is the number of re-implementations of each experiment, performed with the aim of good reproducibility of the experiment result and reduction of variances of measurement errors.

When setting factor experiments use coded values of variables. This greatly facilitates not only the calculation of constant coefficients, but also the study of the subsequently obtained regression model of the response function. Therefore, we pass from the coordinate system of variables $L, B, H$ in the natural dimension (3) to the dimensionless coordinate system. Using the formula [24], convert independent variables into dimensionless variables,

$$
x_{i}=\frac{2\left(\ln x_{l}-\ln x_{\iota \max }\right)}{\ln x_{\imath \max }-\ln x_{\imath \min }}+1
$$

where $\chi_{i}$ is the natural measurement of variables. Moreover, in the dimensionless coordinate system for the model $n^{k}=2^{3}$ of a complete factorial experiment, the upper level corresponds to the value of the factor " +1 " ( $\max$ ), the lower level "-1" ( $\mathrm{min})$, and the coordinates of the center of the experimental. The results of coding the upper and lower levels of variation of factors are summarized in Table 2. From Table 2 we find dimensionless variables,

$$
x_{i}=\frac{2\left(\ln x_{l}-2.9957\right)}{2.9957-1.6094}+1=1.4427 \ln \chi_{l}-3.322
$$

the model $n^{k}=2^{3}=8$ of a complete factorial experiment is presented in the form of an incomplete quadratic polynomial, which can be written taking into account the interaction of factors and in coded variables,

$$
y=b_{0}+b_{1} x_{1}+b_{2} x_{2}+b_{3} x_{3}+b_{12} x_{1} x_{2}+b_{13} x_{1} x_{3}+b_{23} x_{2} x_{3}+b_{123} x_{1} x_{2} x_{3}
$$

where: $b_{0}, b_{i, j, k}$ constant factors for factors: $x_{i, j, k} ; i, j, k=1,2,3$.

In the Table 3 shows the levels of variation of independent factors and their interaction, i.e. the matrix of experimental planning, in accordance with which the variation of each of the factors was performed: $L, B, H$-length, width, height of the mineral at two extreme levels " +1 " (max) and " -1 " (min). 
Table 2. Levels of change of factors

\begin{tabular}{ccccccc}
\hline Levels factors & \multicolumn{2}{c}{$\mathrm{L}(\mathrm{mm})$} & \multicolumn{2}{c}{$\mathrm{B}(\mathrm{mm})$} & \multicolumn{2}{c}{$\mathrm{H}(\mathrm{mm})$} \\
\hline & $\chi_{1}$ & $\ln \hat{\chi}_{1}$ & $\hat{\chi}_{2}$ & $\ln \hat{x}_{2}$ & $\chi_{3}$ & $\ln \chi_{3}$ \\
Upper +1 & 20 & 2.99 & 20 & 2.99 & 20 & 2.99 \\
Basic 0 & 12.5 & - & 12.5 & - & 12.5 & - \\
Lower -1 & 5 & 1.6 & 5 & 1.6 & 5 & 1.6 \\
\hline
\end{tabular}

Table 3. Extreme experiment planning matrix

\begin{tabular}{|c|c|c|c|c|}
\hline \multicolumn{5}{|c|}{ Factors and levels of their variation } \\
\hline № experience & $x_{0}$ The code & $x_{1}$ The code $L_{i}, \mathrm{~mm}$ & $x_{2}$ The code $B_{i}, \mathrm{~mm}$ & $x_{3}$ The code $H_{i}, \mathrm{~mm}$ \\
\hline 1 & + & +20 & +20 & +20 \\
\hline 2 & + & -5 & +20 & +20 \\
\hline 3 & + & +20 & -5 & +20 \\
\hline 4 & + & -5 & -5 & +5 \\
\hline 5 & + & +20 & +20 & -5 \\
\hline 6 & + & -5 & +20 & -5 \\
\hline 7 & + & +20 & -5 & -5 \\
\hline 8 & + & -5 & -5 & -5 \\
\hline \multicolumn{5}{|c|}{ Variation in the interaction of factors } \\
\hline № experience & $x_{1} x_{2}$ The code & $:_{1} x_{3}$ The code & $x_{2} x_{3}$ The code & $x_{1} x_{2} x_{3}$ The code \\
\hline 1 & + & + & + & + \\
\hline 2 & - & - & + & - \\
\hline 3 & - & + & - & - \\
\hline 4 & + & - & - & + \\
\hline 5 & + & - & - & - \\
\hline 6 & - & + & - & + \\
\hline 7 & - & - & + & + \\
\hline 8 & + & + & + & - \\
\hline
\end{tabular}

In the Table 4 shows the results of experimental studies of the influence of parameters, length, width and height of the mineral on the value of the volume $V$ of the mineral stone. The vertical columns show the obtained value of volume $V$, corresponding to the levels of variation of factors, and the horizontal rows contain the results of reproduction of each experiment. The column shows the average values of the volume $V$, and in Table 5 shows their coded values (logarithms) and variances of experimental results.

Table 4. The results of the experiment

\begin{tabular}{cccccccc}
\hline \multicolumn{2}{c}{ Volume $V, \mathrm{~mm}^{3}$ (with the number of experiments $\left.m \rightarrow V_{1} \ldots V_{8} ; y_{i}=\ln V_{i}\right)$} & Averages \\
№ experience & $V_{1}$ & $\left(y_{i}\right)$ & $V_{2}$ & $\left(y_{i}\right)$ & $V_{3}$ & $\left(y_{i}\right)$ & $\bar{V}_{i}$ \\
\hline 1 & 8000 & $(8.99)$ & 6400 & $(8.76)$ & 9920 & $(9.02)$ & 8107 \\
2 & 2000 & $(7.60)$ & 2240 & $(7.71)$ & 1520 & $(7.33)$ & 1920 \\
3 & 216 & $(7.60)$ & 2480 & $(7.82)$ & 1680 & $(7.39)$ & 2053 \\
4 & 490 & $(6.19)$ & 450 & $(6.1)$ & 550 & $(6.3)$ & 496 \\
5 & 300 & $(5.7)$ & 340 & $(5.8)$ & 270 & $(5.6)$ & 303 \\
6 & 2250 & $(7.7)$ & 2050 & $(7.6)$ & 2560 & $(7.8)$ & 2287 \\
7 & 6050 & $(8.7)$ & 5400 & $(8.6)$ & 6300 & $(8.7)$ & 5916 \\
8 & 4500 & $(8.4)$ & 4100 & $(8.3)$ & 4700 & $(8.5)$ & 4433 \\
\hline
\end{tabular}

Table 5. Coded values and variances of experimental results

\begin{tabular}{cccc}
\hline \multirow{2}{N}{$\begin{array}{c}\text { Coded volume values } \\
\text { experience }\end{array}$} & $\bar{y}_{i}=\ln \bar{V}_{i}$ & $\begin{array}{c}\text { Dispersion } \\
\left.\widehat{(y}_{i}-\bar{y}_{i}\right)^{2}\end{array}$ \\
\hline 1 & 9.00 & $\hat{y}_{i}=\frac{\sum_{i}^{n} y_{1}}{n}$ & 0.0064 \\
2 & 7.56 & 8.92 & 0.0484 \\
3 & 7.63 & 7.34 & 0.0009 \\
4 & 6.21 & 7.60 & 0.0004 \\
5 & 5.72 & 6.19 & 0.0004 \\
6 & 7.71 & 5.70 & 0.0001 \\
7 & 8.69 & 7.70 & 0.0009 \\
8 & 8.39 & 8.66 & 0.0001 \\
& \multicolumn{5}{c}{$n$} \\
\end{tabular}


The obtained data allow to determine the numerical values of the constant coefficients in (6). Due to the fact that the full factorial experiment refers to orthogonal plans, the calculation of regression coefficients is greatly simplified. According to the instructions [24] they can be defined as (7).

$$
b_{i}=\sum_{i=1}^{n} \frac{x_{i} \bar{y}_{i}}{m}
$$

where: $i$-experiment number; $\mathrm{m}$ is the number of studied points in the plan; $\bar{y}_{i}$ - the average response to the number of repeated experiments at the corresponding point of the matrix of the experimental plan.

When $i=0$, the coefficient is calculated. When varying each factor at two levels $(+1)$ and $(-1)$, the calculations are reduced to assigning a column of signs $(+,-)$ to the column of the corresponding factor and algebraic addition of the obtained values. The next division of the obtained result by the number of investigated points $(n=8)$ in the plan gives the required coefficient. Calculating, thus, the values of the coefficients and substituting them in (6), we obtain the regression equation. After statistical analysis of the experimental results, which included checking the homogeneity of the variances of the experimental data in Table 4 by Kochren's criterion, checking the significance of the coefficients of the regression equation using Student's t-test, and also the adequacy of the model to the real process (namely, the accuracy of the experimental data) was verified by the well-known Fisher criterion [24]-[27]. The final version of the regression model of the mineral sorting process,

$$
y=7.614+0.146 x_{1}+0.116 x_{2}+0.014 x_{3}
$$

a multiple correlation coefficient was used to estimate the contribution of regression coefficients to the model equation. In this case, the correlation coefficient was $K=0.9987$, so the regression (8) almost completely describes the results of the experiment [28]-[33].

\section{RESULTS AND DISCUSSION}

The (8) in the coded variables establishes a statistical relationship between the parameter of the volume of the mineral $\mathrm{V}$ and the factors, ie the length $L$, width $B$ and height $H$ of the stones of semi-precious minerals, in this case a mineral such as amber. By analyzing the value and sign of each regression coefficient (8), it is possible to obtain reliable information about the influence of factors on the parameter of mineral volume.

\subsection{Investigation of the regression model of the response function}

In (8), the coefficients for variables are positive, therefore, the approximation of the length of the mineral to its width and height in the selection of minerals contributes to a more efficient use of the material of such raw materials as amber. However, the parameter of the height of the mineral at a stable position in the recognition zone as shown in Figure 4, judging by the value of the coefficient, has less effect on the determination of the volume of the mineral. This is due to the fact that in the process of passing the zone of mineral recognition on the belt conveyor, the mineral stones will always be in a stable position under the action of their weight. In other words, the length and width of the mineral stone will dominate. Other coefficients for pair wise interaction as well as triple interaction of factors were excluded as statistically insignificant according to Student's criterion. Note that the influence of interaction effects is manifested only in the combinations of factors and does not apply to the analysis of the influence of each factor separately. The autonomous influence of factors, as noted above, is determined by the magnitude and sign of the coefficients for them. The obtained data indicate the direction of effective sorting of minerals from amber, namely for the manufacture of jewelry should be used stones with similar in size in length, width and height of mineral stones. In this case, there will be a minimum amount of waste during the machining of minerals. For numerical analysis of the influence of factors $x_{1}, x_{2}, x_{3}$ on the useful volume $V\left(\mathrm{~mm}^{3}\right)$ of the mineral, we substitute the coded values of variables (5) in (8) and after potentiation, we obtain the expression of the (1) that reflects the variables $L, B, H$ in natural units $(\mathrm{mm})$.

$$
V=812.4 L^{0.2106} B^{0.1674} H^{0.0202}
$$

In Figure 6 shows graphs of the influence of geometric parameters of minerals on their volumes according to function (9). Each of the graphs $V(L), V(B), V(H)$ shows the change in the volume $V$ of the mineral at the average value of the other parameters. These dependences confirm the dominant influence on the volume $V$ of the mineral of the parameters $L, B$, ie its length and width, which corresponds to the values of the coefficients for these parameters in the regression model (8). If we take the effect on the volume of the 
mineral of the parameter length $L$ for $100 \%$, then when changing the size of the minerals from a minimum value of $5 \mathrm{~mm}$ to a maximum value of $20 \mathrm{~mm}$ as shown in Table 1 , the effect of width $B$ is $79.6 \%$. At the same time, the effect on the volume of the mineral of height $H$ is only $10.2 \%$. When selecting minerals for the manufacture of jewelry, preference should be given to mineral blanks with similar in size to all three parameters. In this case, there will be a minimum amount of mineral waste during their machining. In other words, can make more products from one mineral.

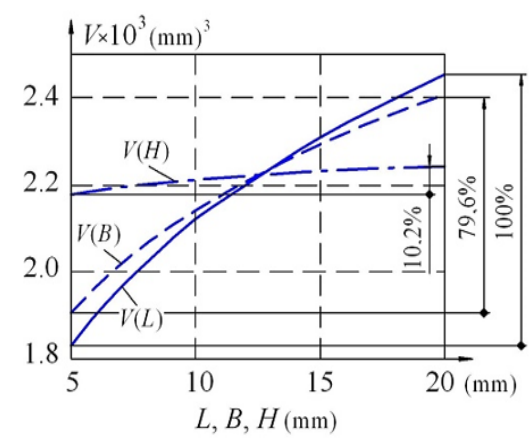

Figure 6. Graphs of the influence of geometric parameters of minerals on their volumes

\subsection{A result of the research}

As a result of the research, an intelligent plant for sorting semi-precious minerals was created. The artificial intelligence [28] of the installation consists of the following automatic operations, namely: 1) determination of the gross volume of the mineral stock; 2) determination of the net volume of future jewelry; 3 ) calculation of the minimum difference between the gross volume of the mineral stock and the net volume of future jewelry; 4) on the basis of the previous operation, that is, determining the minimum difference of the indicated volumes, the distribution of mineral blanks is carried out in the respective containers. Thus, by determining the maximum possible number of finished jewelries from each mineral, it seems possible to reduce the waste of semiprecious minerals.

A number of experiments were carried out according to the classical formula $N=n^{k} m=2^{3} .3=$ 24 realizations, where: $n$ is the number of levels of factor variation ( $\max , \min ) ; k$-is the number of factors; $m$ is the number of re-implementations of each experiment, performed with the aim of good reproducibility of the experiment result and reduction of variances of measurement errors. Mineral volumes were measured in automatic mode using of three charge-coupled device (CCD) matrices for identifying volumes of minerals in a $3 \mathrm{D}$ coordinate system according to the algorithm of the program described above (see section 2.2. The method of minerals recognition) followed by checking with standard measuring instruments with an accuracy of $0.2 \mathrm{~mm}$. A regression model of the mineral sorting process (8) and its analytical expression (9) were obtained. The reliability of the model was verified by the classical Cochran's tests, the student's t-test, as well as the adequacy of the model by the Fisher's test [24], [26].

Tests of the prototype of the installation showed the following results: the identification time of each mineral was $0.8 \ldots 1.2$ seconds, the average productivity of the installation was 3600 units of minerals per hour, which is more than an order of magnitude higher than the productivity of a human inspector. In addition, in the manufacture of spherical beads from amber, the volume of raw waste from $30 \% \ldots 35 \%$, which is typical for a human inspector, decreased to $10 \% \ldots 15 \%$ during operation of the developed installation.

The experimental selection of stones of semiprecious minerals in the form of amber in a volume of more than 1000 pieces shows that most of them have sizes in the range from 10 to $20 \mathrm{~mm}$ as shown in Table 1. And the results of the experiment indicate a dominant effect on the volume of the mineral of its length and width. This is explained by the fact that when minerals move along conveyor 4 as shown in Figure 4 and when they enter the recognition zone with video cameras as shown in pos. 7, Figure 4, the minerals occupy a stable position under the influence of their of weight. A number of possible future studies using the same experimental set up are apparent.

\section{CONCLUSION}

This work indicated that generally, as a result of the research the technology of intelligent sorting of semi-precious minerals was created, which is aimed at reducing raw waste. The method for sorting minerals 
which is using the indicated technology consists of the following: initially, the gross volume of the mineral arriving for sorting is determined, and then it is compared with one or more net volumes of manufactured jewelry and the minimum difference between the indicated volumes is calculated. In other words, the minimum possible amount of unavoidable waste is determined.

The developed installation promotes the rational use of semi precious minerals such as amber, emeralds, garnets, topazes, Icelandic spar, rock crystal and other semi precious minerals. The application of the above technology contributes not only to increase labor productivity in the jewelry industry, but most importantly, it saves raw materials in the form of various semi precious minerals. Ultimately, this result contributes to environmental cleanliness in the extraction of natural resources in the form of semi-precious minerals.

As the results of the experiment show, when sorting semiprecious minerals, preference should be given to minerals that have similar lengths, widths, and their weights. In this case, it is possible to manufacture a greater number of jewelry items with minimal waste. This work has open up several questions that need of further investigation. This helps to increase the profit of jewelry enterprises.

\section{REFERENCES}

[1] B. Ranft and C. Stiller, "The Role of Machine Vision for Intelligent Vehicles," in IEEE Transactions on Intelligent Vehicles, vol. 1, no. 1, pp. 8-19, March 2016, doi: 10.1109/TIV.2016.2551553.

[2] F. Juefei-Xu, D. K. Pal, K. Singh and M. Savvides, "A preliminary investigation on the sensitivity of COTS face recognition systems to forensic analyst-style face processing for occlusions," 2015 IEEE Conference on Computer Vision and Pattern Recognition Workshops (CVPRW), Boston, MA, pp. 25-33, 2015, doi: 10.1109/CVPRW.2015.7301316.

[3] T. S. Musab, H. Abd Al-Raheem Taha, M. Abd Shehab and M. A.M. Abdullah, "Comparison of feature extraction and normalization methods for speaker recognition using grid-audiovisual database," Indonesian Journal of Electrical Engineering and Computer Science (IJEECS), vol. 18, no. 2, pp. 782-789, May 2020, doi: 10.11591/ijeecs.v18.i2.pp782-789, doi: 10.11591/ijeecs.v18.i2.pp782-789.

[4] E. Zancul., H. O.Martins, F.P. Lopes and F.A.T.V.da Silva Neto, "Machine Vision applications in a Learning Factory," Procedia Manufacturing, vol. 45, pp. 516-521, Apr. 2020, doi: 10.1016/j.promfg.2020.04.069.

[5] B. I. Oladapo, et al., "Model design and simulation of automatic sorting machine using proximity sensor," Engineering Science and Technology, an International Journal, vol. 19, no. 3, pp. 1452-1456, Sept. 2016, doi: 10.1016/j.jestch.2016.04.007.

[6] P. Vangla, N. Roy and M.L. Gali, "Image based shape characterization of granular materials and its effect on kinematics of particle motion," Granular Matter, vol. 20, no. 6, Dec. 2018, doi: 10.1007/s10035-017-0776-8.

[7] S. Pellegrinelli, "Configuration and reconfiguration of robotic systems for waste macro sorting," International Journal of Advanced Manufacturing Technology, vol. 102, pp. 3677-3687, Feb. 2019, doi:10.1007/s00170-01903289-x.

[8] Tako P.R. de Jong, "Automatic Sorting of Minerals," IFAC Proceedings Volumes, vol. 37, no. 15, pp. 441-446, Sept. 2004, doi: 10.1016/S1474-6670(17)31064-9.

[9] R. Adamietz, T. Giesen Pablo, M. Andrew, J. Richard and C. Seifartha, "Reconfigurable and transportable container-integrated production system," Robotics and Computer-Integrated Manufacturing, vol. 53, pp. 1-20, Oct. 2018, doi: 10.1016/j.rcim.2018.02.008.

[10] G. Bonifazi and S. Serranti,"Recycling Technologies," In: Themelis N., Bourtsalas A. (eds) Recovery of Materials and Energy from Urban Wastes. Encyclopedia of Sustainability Science and Technology Series. New York, NY: Springer, 2019, doi: 10.1007/978-1-4419-0851-3 116.

[11] Y. Liu, H. Yu, C. Gong and Y. Chen, "A real time expert system for anomaly detection of aerators based on computer vision and surveillance cameras," Journal of Visual Communication and Image Representation, vol. 68, 102767, Apr. 2020, doi: 10.1016/j.jvcir.2020.102767.

[12] R. Biswas, J. Uddin and Md. Junayed Hasan, "A New Approach of Iris Detection and Recognition," International Journal of Electrical and Computer Engineering (IJECE), vo.1 7, no. 5, pp. 2530-2536, Oct. 2020, doi: 10.11591/ijece.v7i5.pp2530-2536.

[13] B. G. Batchelor," Machine Vision for Industrial Applications," In: Batchelor B.G. (eds) Machine Vision Handbook. London: Springer, 2012, doi: 10.1007/978-1-84996-169-1.

[14] I. Parkhomey, J. Boiko and O. Eromenko, "Identification information sensors of robot systems," Indonesian Journal of Electrical Engineering and Computer Science (IJEECS), vol. 14, no. 3, pp. 1235-1243, June 2019, doi: 10.11591/ijeecs.v14.i3.pp1235-1243.

[15] M. Polishchuk, M. Tkach, I. Parkhomey, J. Boiko and O. Eromenko, "Experimental Studies on the Reactive Thrust of the Mobile Robot of Arbitrary Orientation," Indonesian Journal of Electrical Engineering and Informatics, vol. 8, no. 2, pp. 340-352, June 2020, doi: 10.11591/ijeei.v8i2.1681.

[16] S. Boudra, N.E. Berrached and A. Dahane, "Efficient and secure real-time mobile robots cooperation using visual servoing," International Journal of Electrical and Computer Engineering (IJECE), vol. 10, no. 3, pp. 3022-3034, June2020, doi: 10.11591/ijece.v10i3.pp3022-3034. 
[17] A. Nayak and K. Dutta, "Impacts of machine learning and artificial intelligence on mankind," 2017 International Conference on Intelligent Computing and Control (I2C2), Coimbatore, 2017, pp. 1-3, doi: 10.1109/I2C2.2017.8321908.

[18] M. M. Polishchuk, "The method of sorting small minerals and device for its implementation," UA Patent 116863, May 10. 2018.

[19] H. N. Mohd Shah, et al., "Develop and implementation of PC based controller for humanoid robot using digital potentiometer," Indonesian Journal of Electrical Engineering and Computer Science (IJEECS), vol. 15, no. 1, pp. 104-112, July 2019, doi: 10.11591/ijeecs.v15.i1.pp104-112.

[20] J. Han, et al., "Research on Carved Turns of a Skiing Humanoid Robot on a Real-World Slope," 2018 IEEE/RSJ International Conference on Intelligent Robots and Systems (IROS), Madrid, pp. 1-9, 2018, doi: 10.1109/IROS.2018.8593796.

[21] T. Tang, T. Yang, B. Qi, G. Ren and Q. L. Bao, "Error-Based Feedforward Control for a Charge-Coupled Device Tracking System," in IEEE Transactions on Industrial Electronics, vol. 66, no. 10, pp. 8172-8180, Oct. 2019, doi: 10.1109/TIE.2018.2884239.

[22] S. Shabaev, N. Martel, A. Shtark and E. Rysbekova, "Improving the method for determining the granular media strength performance by oblique shear test," E3S Web of Conferences, vol. 174, 01064, June 2020, doi: $10.1051 / \mathrm{e} 3$ sconf $/ 202017401064$.

[23] E. Gülcan and Ö. Y. Gülsoy, "Performance evaluation of optical sorting in mineral processing-A case study with quartz, magnesite, hematite, lignite, copper and gold ores," International Journal of Mineral Processing, vol. 169, pp. 129-141, Dec. 2019, doi: 10.1016/j.minpro.2017.11.007.

[24] I.I. Plyaskin, "Optimization of technical solutions in mechanical engineering," Moskva M: Mechanical Engineering, 1982. 176p.

[25] I. Parkhomey, J. Boiko, N. Tsopa, I. Zeniv and O. Eromenko, "Assessment of quality indicators of the automatic control system influence of accident interference," TELKOMNIKA (Telecommunication Computing Electronics and Control), vol. 18, no. 4, pp. 2070-2079, Aug. 2020, doi: 10.12928/telkomnika.v18i4.15601.

[26] T. Halme, V. Koivunen and H. V. Poor, "Nonparametric distributed detection using bootstrapping and fisher's method," 2018 52nd Annual Conference on Information Sciences and Systems (CISS), Princeton, NJ, pp. 1-6, 2018, doi: 10.1109/CISS.2018.8362316.

[27] Y. Li, W. Zhao and J. Pan, "Deformable Patterned Fabric Defect Detection with Fisher Criterion-Based Deep Learning," in IEEE Transactions on Automation Science and Engineering, vol. 14, no. 2, pp. 1256-1264, April 2017, doi: 10.1109/TASE.2016.2520955.

[28] M. S. Gaya, et al., "Estimation of water quality index using artificial intelligence approaches and multi-linear regression," IAES International Journal of Artificial Intelligence (IJ-AI), vol. 9, no. 1, pp. 126-134, March 2020, doi: 10.11591/ijai.v9.i1.pp126-134.

[29] Y. Lin, H. Min, H. Zhou and F. Pei, "A Human-Robot-Environment Interactive Reasoning Mechanism for Object Sorting Robot," in IEEE Transactions on Cognitive and Developmental Systems, vol. 10, no. 3, pp. 611-623, Sept. 2018, doi: 10.1109/TCDS.2017.2706975.

[30] J. Boiko, I. Pyatin, O. Eromenko and O. Barabash, "Methodology for Assessing Synchronization Conditions in Telecommunication Devices," Advances in Science, Technology and Engineering Systems Journal, vol. 5, no.2, pp. 320-327, March 2020, doi: 10.25046/aj050242.

[31] S. Ibrahim, N. Amirah Zulkifli, N. Sabri, A. A. Shari and M. R. Mohd Noordin, "Rice grain classification using multi-class support vector machine (SVM)," IAES International Journal of Artificial Intelligence (IJ-AI), vol. 8, no. 3, pp. 215-220, Sept. 2019, doi: 10.11591/ijai.v8.i3.pp215-220.

[32] D. Nandkishor, C. N. Raju and D. A. Ramchandra, "Preprogramming Enabled Lego Robot and its Use in Robotics Education," gis.Science-Die Zeitschrift fur Geoinformatik, vol. 7, no. 11, pp. 1393-1399, Nov. 2020, doi: 20.18001.GSJ.2020.V7I11.20.36077.

[33] J. Boiko, L. Karpova, O. Eromenko and Y. Havrylko, "Evaluation of phase-frequency instability when processing complex radar signals," International Journal of Electrical and Computer Engineering (IJECE), vol. 10, no. 4, pp. 4226-4236, Aug 2020, doi: 10.11591/ijece.v10i4.pp4226-4236. 